\title{
A Binary Supramolecular Assembly with Intense Fluorescence Emission, High pH Stability, and Cation Selectivity: Supramolecular Assembly-Induced Emission Materials
}

\author{
Xu Wang ${ }^{1}$, Xin-Yue Lou ${ }^{1}$, Xiao-Yu Jin ${ }^{1}$, Feng Liang ${ }^{2}$, and Ying-Wei Yang ${ }^{1,2}$ \\ ${ }^{1}$ State Key Laboratory of Inorganic Synthesis and Preparative Chemistry, International Joint Research Laboratory of \\ Nano-Micro Architecture Chemistry, College of Chemistry, Jilin University, 2699 Qianjin Street, Changchun 130012, China \\ ${ }^{2}$ The State Key Laboratory of Refractories and Metallurgy, School of Chemistry \& Chemical Engineering, Wuhan University of \\ Science and Technology, Wuhan 430081, China
}

Correspondence should be addressed to Ying-Wei Yang; ywyang@jlu.edu.cn

Received 30 April 2019; Accepted 17 June 2019; Published 24 July 2019

Copyright ( 2019 Xu Wang et al. Exclusive Licensee Science and Technology Review Publishing House. Distributed under a Creative Commons Attribution License (CC BY 4.0).

We construct a fluorescent supramolecular system (TPE- $\left.\mathrm{Q}_{4} \subset \mathrm{DSP} 5\right)$ of excellent tolerance to a wide range of $\mathrm{pH}$ by the facile selfassembly of a new pillar[5] arene bearing disulfonated arms (DSP5) with an AIE-active tetraphenylethene-based tetratopic guest bearing four quaternary ammonium binding sites $\left(\mathrm{TPE}-\mathrm{Q}_{4}\right)$, which exhibits strong blue emission even in dilute aqueous solutions along with much higher quantum yield and longer fluorescence lifetime than TPE- $\mathrm{Q}_{4}$ itself. This appreciable property can be attributed to the supramolecular assembly-induced emission (SAIE) mechanism endowed by the host-guest inclusion complexation based on synthetic macrocycles. Remarkably, the enhanced fluorescence of the supramolecular assembly is quenched efficiently and exclusively by ferric ions in water with a high Stern-Volmer formula constant of $1.3 \times 10^{5} \mathrm{~mol}^{-1}$, demonstrating the excellent cation selectivity and visualized responsiveness in ion sensing and detection.

\section{Introduction}

Fluorescent supramolecular assemblies represent an extraordinarily attractive class of functional systems, due to their integrated functionalities, highly tunable fluorescence properties, and good responsiveness towards external stimuli [1]. The inherent features of their fluorescent entities that can be further mediated by the rational design of supramolecular building blocks endow them extensive potentials in smart materials and light-emitting devices [2]. In 2001, Tang and coworkers discovered some propeller-like molecules with $\pi$-conjugated structures that exhibited weak fluorescence in solution but bright emission in aggregated states in sharp contrast to traditional fluorescent molecules of aggregation caused quenching (ACQ) and creatively named this phenomenon aggregation-induced emission (AIE) [3]. Many fluorophores with AIE properties (AIEgens) have been discovered and extensively investigated for applications in chemistry and materials science $[4,5]$. The mechanism of fluorescent emission enhancement of AIEgens in the aggregated states mainly lies in the restriction of intramolecular motion
(RIM) and the blockade of nonradiation energy dissipation channel from the excited state to ground state $[4,5]$.

Inspired by the mechanism of AIE, researchers have successfully developed a supramolecular pathway, namely, supramolecular assembly-induced emission (SAIE), to achieve effective emission enhancement of AIEgens in the ordered assembly states no matter in dilute solutions or aggregated solid states [2, 6-19]. In 2014, Tang and coworkers covalently linked a cyclodextrin with tetraphenylethene (TPE) and utilized self-inclusion complexation for RIM to achieve enhanced emission in aqueous solutions, which represented the prototype SAIE study and paved the way of recruiting supramolecular macrocycles to manipulate the fluorescent properties of AIEgens [6]. Considerable effort has been made in the rapid development of SAIE recently, accompanied by the synthesis of functional supramolecular assemblies with tunable emission [7-19]. Various types of supramolecular fluorescent assemblies based on AIEgens and functional synthetic macrocycles have been reported for applications in cell imaging, [11] fluorescent supramolecular polymers and gels $[7-10,13,14]$, 


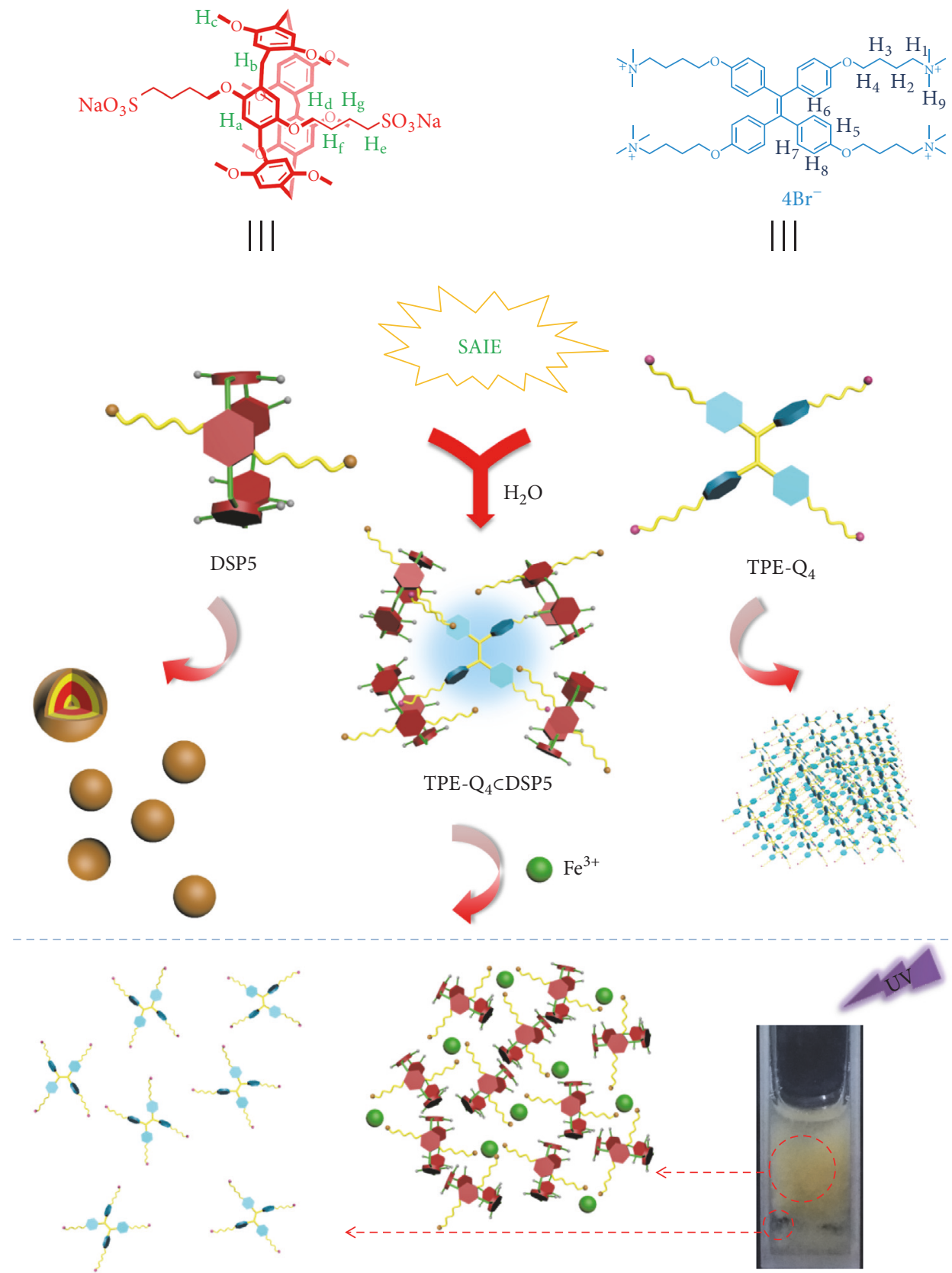

Scheme 1: Chemical structures and cartoons of DSP5 and TPE- $\mathrm{Q}_{4}$ and the schematic presentation of their self-assembly into a fluorescent supramolecular system for the selective detection of $\mathrm{Fe}^{3+}$ ions.

room-temperature phosphorescence [15], controlled drug delivery [16], and other stimuli-responsive materials [1719]. But nonetheless, challenges in their preparation and application remain.

On the other hand, pillarenes, as an important class of macrocyclic hosts in the realm of supramolecular chemistry and advanced functional materials, are attracting more and more attention due to the unique pillar-like rigid structure, electron-rich cavity, well-studied host-guest properties, and facile modification methodology [20-22]. Functionalized pillarenes have played important roles in ion detection [23], antibacterial therapeutics [24], supramolecular nanovalves
[25-29], and so on [30]. Significantly, by elaborate functionalization, pillarenes have been utilized as an important candidate of synthetic macrocycles in the construction of stimuliresponsive fluorescent materials through the marriage with AIEgens [2].

Herein, we report on the fabrication of a $\mathrm{pH}$-tolerant fluorescent system for specific cation recognition based on supramolecular self-assembly of disulfonated pillar[5] arene (DSP5) and a water-soluble TPE-based tetratopic guest (TPE- $\mathrm{Q}_{4}$ ) with four quaternary ammonium binding sites (Scheme 1). Because of the high binding affinity of DSP5 towards $\mathrm{TPE}-\mathrm{Q}_{4}$, a supramolecular assembly $\left(\mathrm{TPE}-\mathrm{Q}_{4} \quad \mathrm{C}\right.$ 
DSP5) was fabricated in aqueous solution via host-guest interaction, exhibiting bright blue emission in a wide range of $\mathrm{pH}$ conditions. Meanwhile, this supramolecular assembly shows a specific response of fluorescence quenching to $\mathrm{Fe}^{3+}$ through the coordination between the sulfonate arms of DSP5 and $\mathrm{Fe}^{3+}$ ions, endowing the material with smart cation responsiveness.

\section{Results}

2.1. Host-Guest Complexation. DSP5 was successfully designed and synthesized for the first time via a Williamson ether-type synthetic approach (Scheme S1). To investigate the host-guest property of DSP5 toward TPE- $\mathrm{Q}_{4}$, the following experiments have been conducted. A TPE-based monotopic guest (TPE-Q) with single quaternary ammonium binding site was chosen as a model guest for the study of the host-guest binding between DSP5 and the guest linker moiety on TPE in deionized water at $298 \mathrm{~K}$ via fluorescence titration (Figure S1). The stoichiometric ratio of DSP5 and the monotopic model guest TPE-Q was determined to be 1:1 and their association constant $\left(K_{a}\right)$ was calculated to be $(2.68 \pm 0.24) \times 10^{5} \mathrm{M}^{-1}$. The $2 \mathrm{D}$ NOESY spectrum (Figures S2 and S3) of the 4:1 mixture of DSP5 (4.0 mM) and $\mathrm{TPE}-\mathrm{Q}_{4}(1.0 \mathrm{mM})$ in $\mathrm{D}_{2} \mathrm{O}$ showed obvious correlations between the phenyl protons $\mathrm{H}_{\mathrm{a}}$ of DSP5 and the alkyl chains protons $\mathrm{H}_{1}-\mathrm{H}_{4}$ and methyl protons $\mathrm{H}_{9}$ of TPE- $\mathrm{Q}_{4}$, indicating the existence of strong host-guest interactions between DSP5 and TPE- $\mathrm{Q}_{4}$ and the inclusion of alkyl chains of TPE- $Q_{4}$ into the cavities of DSP5 macrocycles. Furthermore, DOSY experiments were employed to investigate the formation of TPE-Q 4 C DSP5 in water (see Figure S4). The measured weight-average diffusion coefficient $(D)$ of TPE- $\mathrm{Q}_{4}$ decreased from $9.66 \times 10^{-10}$ to $8.85 \times 10^{-10} \mathrm{~m}^{2} / \mathrm{s}$ with the addition of 4 equiv. of DSP5, suggesting the formation of supramolecular assemblies. Experimental evidence given above has demonstrated the successful construction of the 1:4 supramolecular inclusion complex of TPE- $\mathrm{Q}_{4} \subset \mathrm{DSP} 5$.

\subsection{Fluorescent Properties and Morphology. Fluorescent} changes of TPE-Q ${ }_{4}(1 \mu \mathrm{M})$ in water in the presence of different concentrations of DSP5 were monitored by fluorescence spectroscopy (Figure 1). The emission of TPE- $\mathrm{Q}_{4}$ solution is negligible; however upon addition of DSP5, the whole system exhibited green emission color and the fluorescence intensity dramatically increased, reaching the maxima when 4 eq. of DSP5 was added ([DSP5]/[TPE-Q 4$]=4: 1)$ (Figure $1(\mathrm{a}))$. The rational explanation is that the intramolecular rotation of phenyl rings of TPE- $\mathrm{Q}_{4}$ was restricted by DSP5 upon hostguest inclusion complexation and supramolecular assembly (SAIE). The maximum fluorescence enhancement at the hostguest molar ratio of 4:1 indicated a complete formation of TPE-Q 4 C DSP5; thus this ratio was chosen for the following studies. Additional supporting data including UVVis absorption spectra have also been obtained (Figure S5). It is worth mentioning that negligible changes (Figure 1(b)) could be observed in the fluorescence intensity of TPE- $Q_{4} \subset$
DSP5 over a wide range of $\mathrm{pH}$ (3-13), proving its great $\mathrm{pH}$ tolerance and system robustness.

To further investigate the emissive characteristics of TPE$\mathrm{Q}_{4} \subset \mathrm{DSP} 5$, time-resolved fluorescence data of TPE- $\mathrm{Q}_{4}$ and TPE- $Q_{4} \subset$ DSP5 were collected (Figures 1(c) and 1(d), Table $\mathrm{S} 1)$. The absolute fluorescence quantum yield of $\mathrm{TPE}_{-} \mathrm{Q}_{4} \subset$ DSP5 was $54.40 \%$, which is much higher than that of bare $\mathrm{TPE}^{-\mathrm{Q}_{4}}(0.68 \%)$. The photoluminescence decay profiles of TPE- $Q_{4}$ and TPE- $Q_{4} \subset$ DSP5 suggested that the fluorescence lifetime of TPE- $Q_{4}$ itself is 1.14 ns, while the excited state of TPE-Q $\mathrm{Q}_{4} \subset \mathrm{DSP} 5$ relaxed mainly through a slow pathway with a lifetime of 5.64 ns. Interestingly, TPE- $Q_{4} \subset$ DSP5 showed one order of magnitude smaller nonradiative decay rate constant $\left(8.08 \times 10^{7} \mathrm{~s}^{-1}\right)$ and ca. 16 times bigger radiative decay rate constant $\left(9.64 \times 10^{7} \mathrm{~s}^{-1}\right)$ as compared to bare TPE$\mathrm{Q}_{4}\left(8.68 \times 10^{8} \mathrm{~s}^{-1}\right.$ and $5.90 \times 10^{6} \mathrm{~s}^{-1}$, respectively) (Table S1). These experimental results further support the mechanism of SAIE in the system that the intramolecular motions of the TPE- $\mathrm{Q}_{4}$ units are restricted by DSP5 macrocycles, which block the nonradiative relaxation pathway and populated excitons that underwent radiative decay. The above results evidenced that TPE- $Q_{4} \subset$ DSP5 can emit strong fluorescence even in a dilute solution and DSP5 synthetic macrocycle plays a crucial role in the fluorescence enhancement of this supramolecular assembled system.

Based on the aforementioned knowledge, we deduced that the amphiphilic nature of TPE- $Q_{4}$ would lead to its selfassembly in aqueous solution, while the ensembles might undergo observable alteration by the host-guest complexation upon addition of DSP5. To obtain direct evidence, the size and morphology of the supramolecular assemblies were studied by scanning electron microscope (SEM) and dynamic light scattering (DLS). Interestingly, cube-shaped assemblies of $\mathrm{TPE}_{-} \mathrm{Q}_{4}$ with a diameter of $\sim 300 \mathrm{~nm}$ and spherical micelles of DSP5 with a diameter of $\sim 20 \mathrm{~nm}$ were observed, respectively (Figures 2(a1), 2(a2), 2(b1), and 2(b2)). However, as we envisioned, TPE-Q ${ }_{4} \subset$ DSP5 exhibited bulky cube-like morphology, yet it possessed a larger diameter of $500 \mathrm{~nm}$ as compared with the nanostructures formed by TPE$\mathrm{Q}_{4}$ or DSP5 alone (Figures 2(c1) and 2(c2)). The formation of supramolecular host-guest complex between DSP5 and TPE- $Q_{4}$ affected the overall amphiphilic nature including the size of building blocks (TPE-Q ${ }_{4} \subset$ DSP5 versus TPE- $\mathrm{Q}_{4}$ or DSP5) and introduced the inter-building block electrostatic repulsions, simultaneously contributing to the increased size of the assembled architectures.

2.3. Selective Detection of $\mathrm{Fe}^{3+}$ Ion. Inspired by the above advances, fluorescence experiments were conducted to investigate the responsiveness of TPE- $Q_{4} \subset$ DSP5 toward different analytes such as ions. Fluorescence quenching degrees and the Stern-Volmer formula constants of TPE-Q 4 C DSP5 upon addition of different cations and anions at the same concentration were provided (Figures 3(a) and 3(b)), with $\mathrm{Cl}^{-}$ and $\mathrm{Na}^{+}$as the counterions, respectively. Surprisingly, only $\mathrm{Fe}^{3+}$ ions caused a remarkable fluorescence quenching (1 $\left.I / I_{0}=0.84\right)$ with a considerably high Stern-Volmer formula constant $\left(K_{s v}=2.7 \times 10^{5} \mathrm{~mol}^{-1}\right)$. To further confirm this 

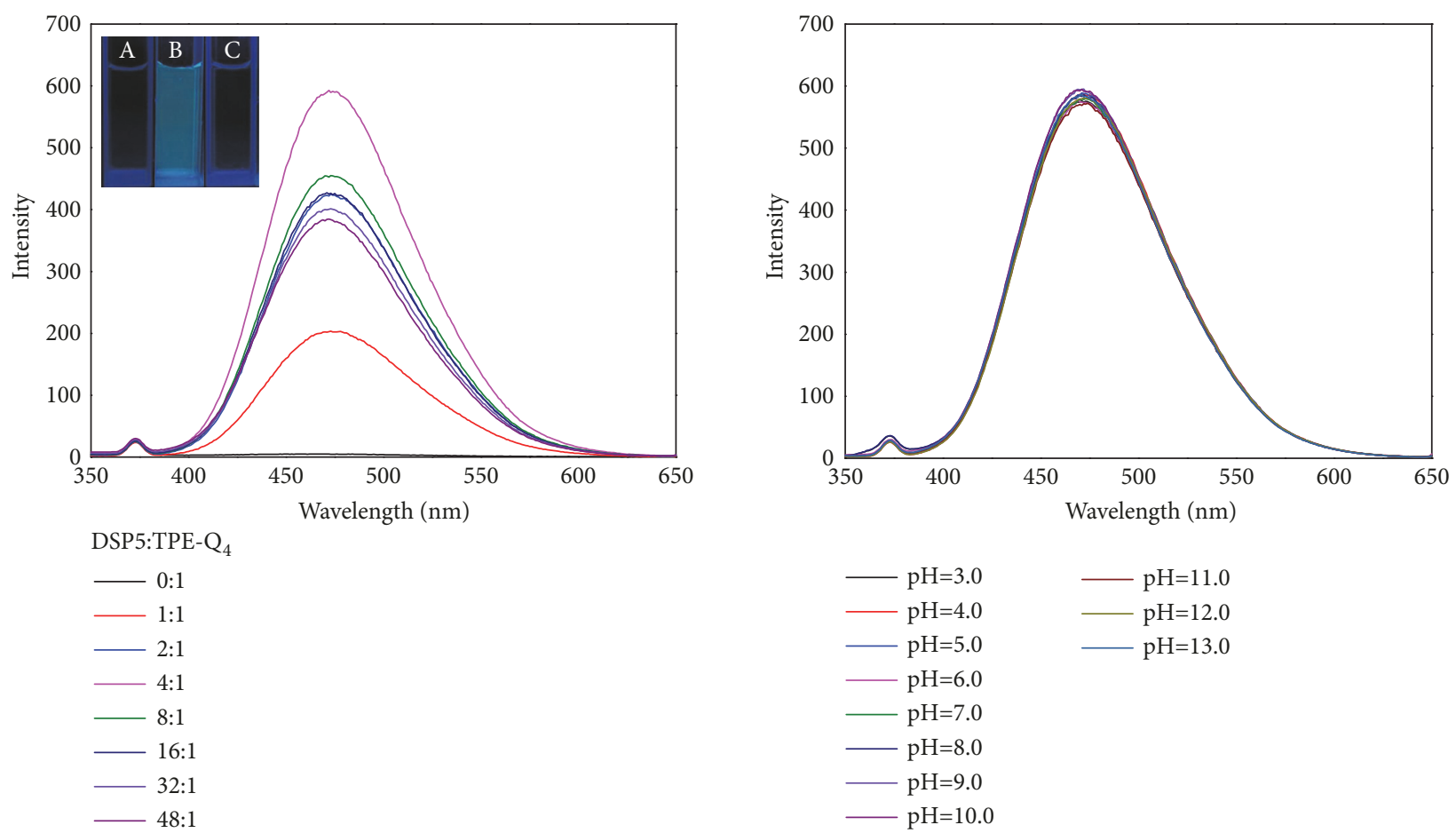

(a)

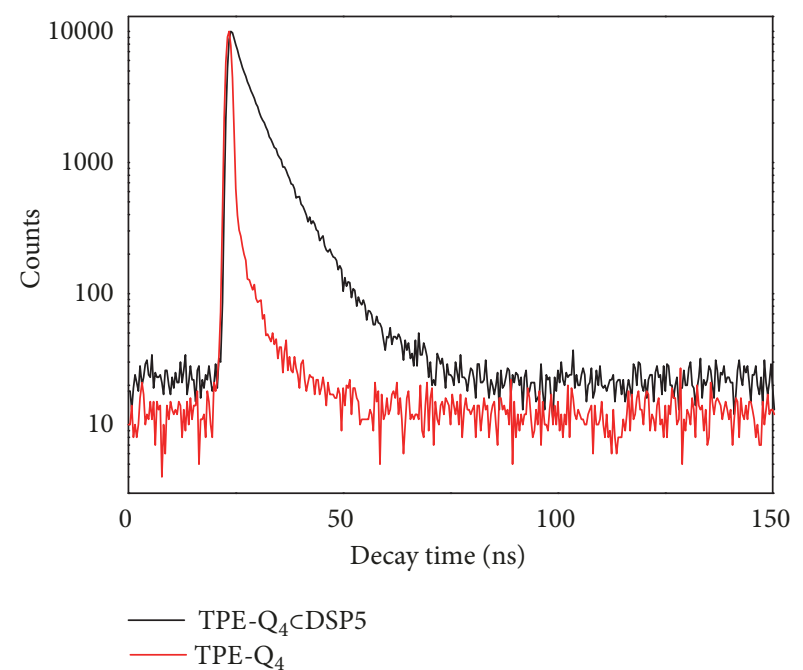

(c)

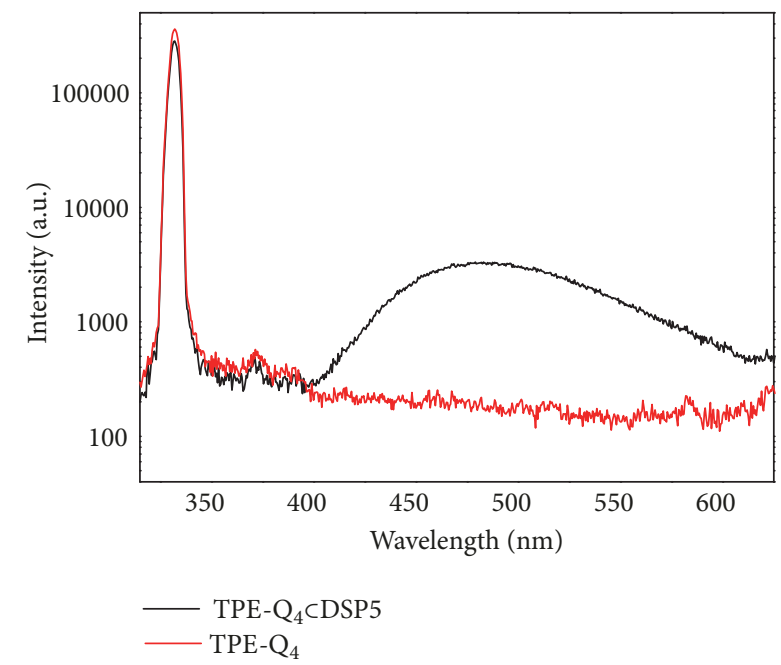

(d)

FIGURE 1: Fluorescence characterizations of TPE- $\mathrm{Q}_{4}$ and TPE- $\mathrm{Q}_{4} \subset$ DSP5. (a) Fluorescence spectra of TPE- $\mathrm{Q}_{4}(1 \mu \mathrm{M})$ in water with different

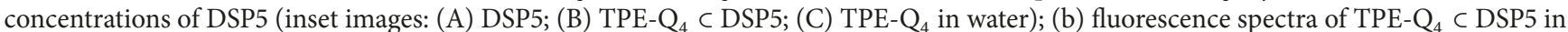
PBS of different pH values. Experimental conditions: $\lambda_{\mathrm{ex}}=330 \mathrm{~nm} ; \lambda_{\mathrm{em}}=475 \mathrm{~nm}$; slit widths: Ex. $5 \mathrm{~nm}, \mathrm{Em} .5 \mathrm{~nm} ; 298 \mathrm{~K} ;\left[\mathrm{TPE}-\mathrm{Q}_{4}\right]=1 \mu \mathrm{M}$, $[D S P 5]=4 \mu \mathrm{M}$. (c) Fluorescence decay profiles and (d) absolute fluorescence quantum yields of TPE- $\mathrm{Q}_{4}$ and TPE-Q ${ }_{4} \subset$ DSP5. Experimental conditions: $\lambda_{\mathrm{ex}}=365 \mathrm{~nm}, 298 \mathrm{~K},\left[\mathrm{TPE}-\mathrm{Q}_{4}\right]=1 \mu \mathrm{M}$, [DSP5] $=4 \mu \mathrm{M}$ in water.

ion sensing specificity, experiments on competitive cations were performed and the results showed that fluorescence quenching degrees are as high $\left(\sim 1-I / I_{0}>0.75\right)$ with or without competition cations (Figure S6). The limit of detection (LOD) of TPE- $\mathrm{Q}_{4} \subset \mathrm{DSP} 5$ to $\mathrm{Fe}^{3+}$ was calculated to be $8.6 \times 10^{-7} \mathrm{M}$ on the basis of $3 \delta / S$, indicating that the material possesses a highly sensitive responsiveness to $\mathrm{Fe}^{3+}$ (Figure S8). Considering that the material exhibits enhanced fluorescence with good $\mathrm{pH}$ stability, the detection of $\mathrm{Fe}^{3+}$ by $T P E-Q_{4} \quad C$ DSP5 was further testified under varied $\mathrm{pH}$ circumstances, and efficient fluorescence quenching was observed within the wide $\mathrm{pH}$ range in the presence of $\mathrm{Fe}^{3+}$ 


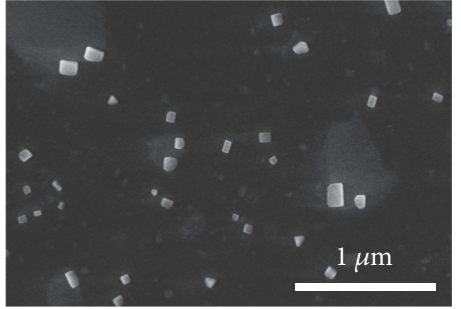

(a1)

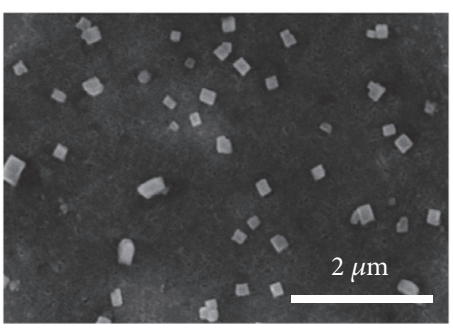

$(\mathrm{c} 1)$

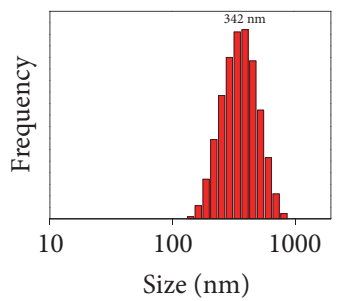

(a2)

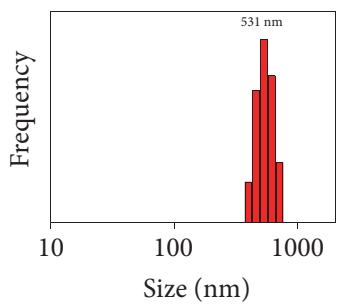

(c2)

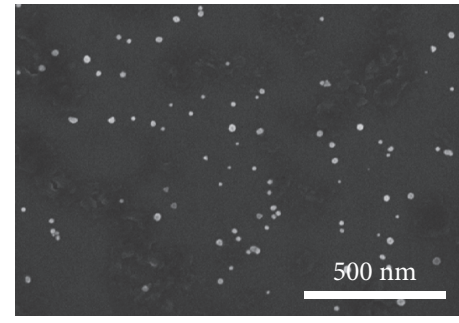

(b1)

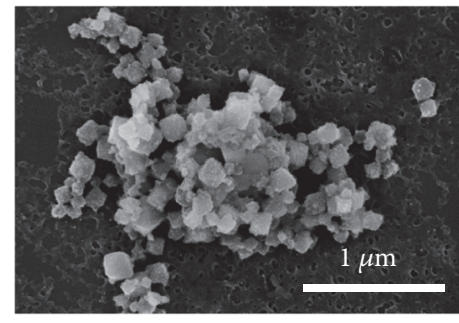

(d1)

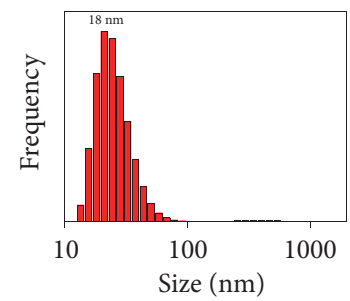

(b2)

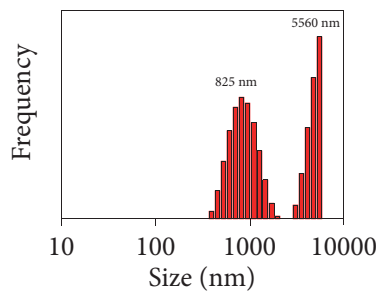

(d2)

Figure 2: Morphology and size characterizations. SEM images and DLS measurements of (a1) and (a2) TPE-Q ${ }_{4}$, (b1) and (b2) DSP5, (c1) and (c2) TPE-Q ${ }_{4} \subset$ DSP5, and (d1) and (d2) TPE-Q 4 C DSP5 with the addition of Fe ${ }^{3+}\left(\left[\mathrm{TPE}-\mathrm{Q}_{4}\right]=1 \mu \mathrm{M},[\mathrm{DSP} 5]=4 \mu \mathrm{M}\right)$.

(Figure S9), which denoted that the material can be applied for the selective detection of $\mathrm{Fe}^{3+}$ in aqueous conditions without the interference of $\mathrm{pH}$ levels.

2.4. Investigation of the Quenching Mechanism. To further explore the quenching mechanism, a series of measurements were carried out. SEM image of TPE- $\mathrm{Q}_{4}$ C DSP5 in the presence of $\mathrm{Fe}^{3+}$ showed irregular morphology, which was different from the assembly structure of TPE- $Q_{4} \subset$ DSP5 itself (Figures 2(d1) and 2(d2)); thus we consider that a complexation between DSP5 and $\mathrm{Fe}^{3+}$ occurred. In order to exclude the influence of the electron-rich cavity of DSP5, monomer molecule (DSNa) with sulfonate groups was synthesized. SEM study demonstrated that $\mathrm{Fe}^{3+}$ could coordinate with the sulfonate groups of DSNa (Figure S10). Moreover, ${ }^{1} \mathrm{H}$ NMR experiments were carried out to confirm the coordination of DSP5 and $\mathrm{Fe}^{3+}$ (Figure S11). Because TPE- $\mathrm{Q}_{4} \subset \mathrm{DSP} 5$ precipitated from $\mathrm{D}_{2} \mathrm{O}$ in the NMR tube, no peaks could be observed in the ${ }^{1} \mathrm{H}$ NMR spectrum. However, the proton peaks of TPE- $\mathrm{Q}_{4}$ reappeared after the addition of $\mathrm{Fe}^{3+}$ but the proton peaks of DSP5 were still absent in the spectrum, suggesting that DSP5 coordinated with $\mathrm{Fe}^{3+}$, forming waterinsoluble coordination polymers. Fluorescent images were also obtained to further confirm the existence of TPE- $\mathrm{Q}_{4}$ in the aqueous media, where the fluorescence recovered when DSP5 was added after the precipitation (Figure S12).

The ion-responsive property of TPE-Q ${ }_{4} \quad$ DSP5 and the interactions of DSP5 and $\mathrm{Fe}^{3+}$ were described in a more intuitive way in Figure 3(c). The TPE- $\mathrm{Q}_{4}$ C DSP5 water solution (B) was clear and emitted intensely under $365 \mathrm{~nm}$ irradiation, yet precipitation and quenching occurred after adding $\mathrm{Fe}^{3+}(\mathrm{C})$ at a higher concentration. After centrifugation, the precipitates were separated from the solutions
(Figure S13). However, A and B solutions still showed Tyndall effect but $\mathrm{C}$ and $\mathrm{D}$ solutions did not, indicating larger supramolecular assemblies in $\mathrm{C}$ and $\mathrm{D}$ than in $\mathrm{A}$ and $\mathrm{B}$ solutions. Importantly, the precipitation in $\mathrm{D}$ further confirmed that DSP5 interacted with $\mathrm{Fe}^{3+}$, leaving TPE- $\mathrm{Q}_{4}$ free in water, where the fluorescence was greatly quenched.

To prove the coordination effect between sulfonate groups of DSP5 and $\mathrm{Fe}^{3+}$, we used X-ray photoelectron spectroscopy (XPS) to record the valence state changes of DSP5 before and after the addition of $\mathrm{Fe}^{3+}$ (Figures 4(a)-4(d)). The XPS spectra demonstrated that the binding energies of the C1s peaks at $284.7 \mathrm{eV}, 290.0 \mathrm{eV}$, and $286.2 \mathrm{eV}$ underwent no obvious changes. But the binding energy of the S2p peak at $168.4 \mathrm{eV}$, attributing to the sulfonate group, changed to 168.8 $\mathrm{eV}$ upon the addition of $\mathrm{Fe}^{3+}$ [31]. Moreover, the binding energy of $\mathrm{Fe} 2 \mathrm{p}(712.2 \mathrm{eV})$ is different from the ferric chloride (711.5 eV) according to previous literature [32]. Besides, solidstate CP-MAS ${ }^{13} \mathrm{C}$ NMR of the DSP 5 and DSP $5+\mathrm{Fe}^{3+}$ powder also provided convincing evidences for the coordination of DSP5 and $\mathrm{Fe}^{3+}$ (Figure 4(e)).

\section{Discussion}

In conclusion, we have successfully synthesized a novel watersoluble disulfonate-functionalized pillar[5] arene (DSP5), based on which a $\mathrm{pH}$-stable fluorescent material (TPE$\mathrm{Q}_{4}$ C DSP5) was facilely designed and constructed, exhibiting SAIE properties. The as-prepared supramolecular material showed excellent photoluminescent characteristics of emitting switchable bright blue fluorescence even in dilute aqueous solution. In addition, the supramolecular assembly possessed superior responsiveness to $\mathrm{Fe}^{3+}$ in water with high selectivity and sensitivity, since the enhanced fluorescence arising from TPE- $\mathrm{Q}_{4} \subset$ DSP5 could be efficiently quenched 


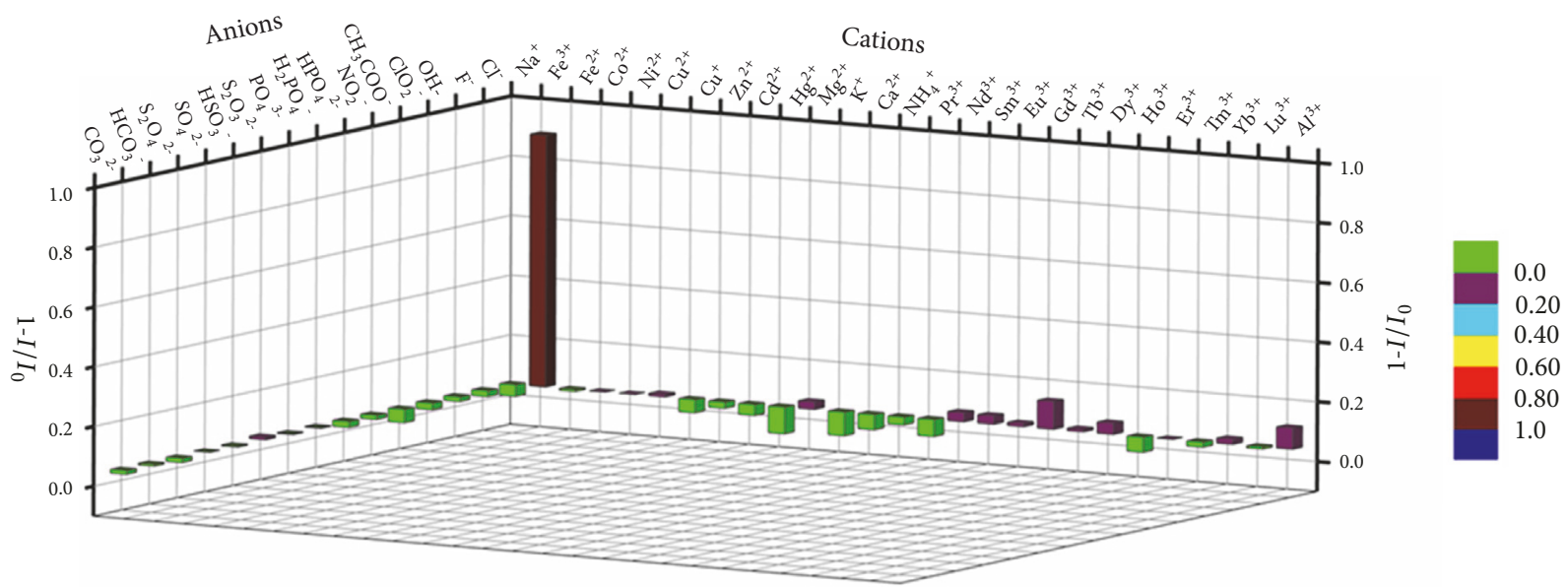

(a)

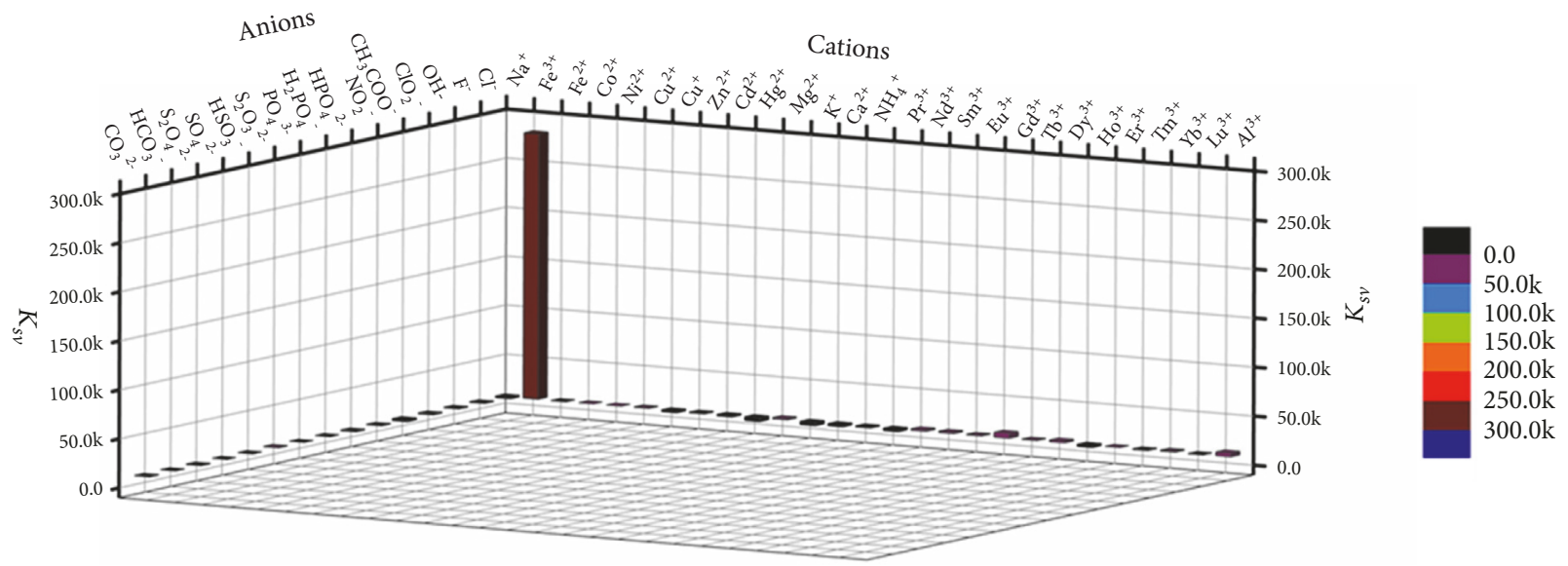

(b)
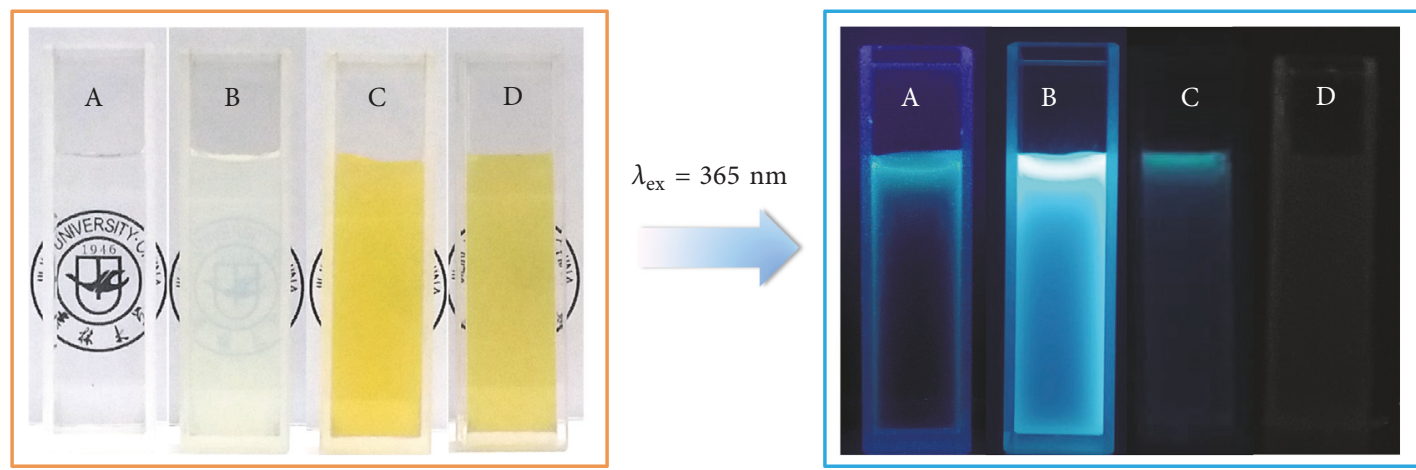

(c)

Figure 3: Highly selective detection of $\mathrm{Fe}^{3+}$ by TPE- $\mathrm{Q}_{4} \subset$ DSP5. (a) Fluorescence quenching degrees of TPE- $\mathrm{Q}_{4} \subset \mathrm{DSP} 5$ in the presence of different cations and anions in water. (b) The Stern-Volmer formula constants of TPE- $Q_{4} \subset$ DSP5 upon interacting with different cations and anions in water. (c) The pictures of (A) TPE-Q 4 , (B) TPE- ${ }_{4} \subset$ DSP5, (C) TPE- $\mathrm{Q}_{4} \subset \mathrm{DSP} 5+\mathrm{Fe}^{3+}$, and (D) DSP5 + Fe $\mathrm{F}^{3+}$ in water. Experimental conditions: $\lambda_{\mathrm{ex}}=330 \mathrm{~nm} ; \lambda_{\mathrm{em}}=475 \mathrm{~nm}$; slit widths: Ex. $5 \mathrm{~nm}, \mathrm{Em} .5 \mathrm{~nm} ; 298 \mathrm{~K}$; [TPE-Q $\left.{ }_{4}\right]=1 \mu \mathrm{M}$, [DSP5] $=4 \mu \mathrm{M},\left[\mathrm{Fe}^{3+}\right]=20 \mu \mathrm{M}$, [Ions] $=$ $20 \mu \mathrm{M}$. 


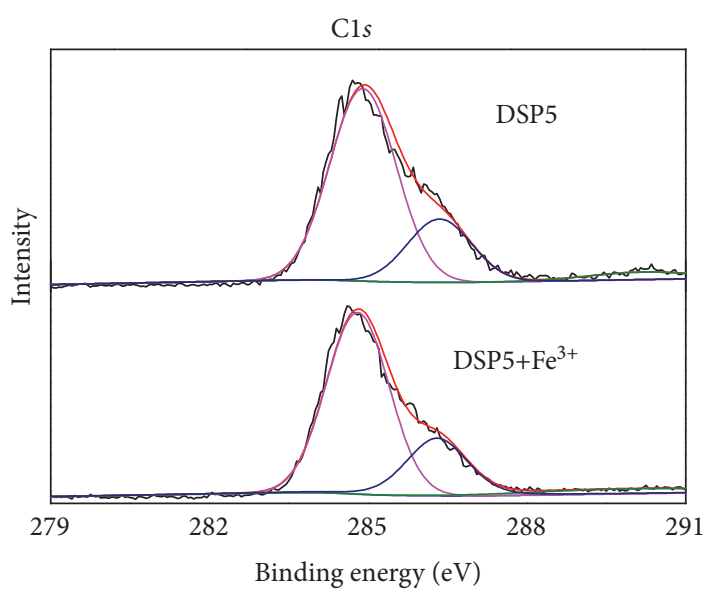

(a)

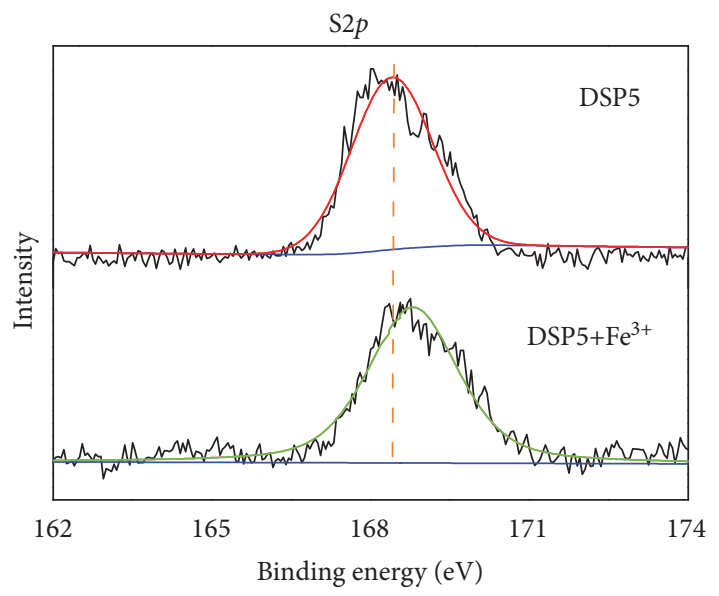

(c)

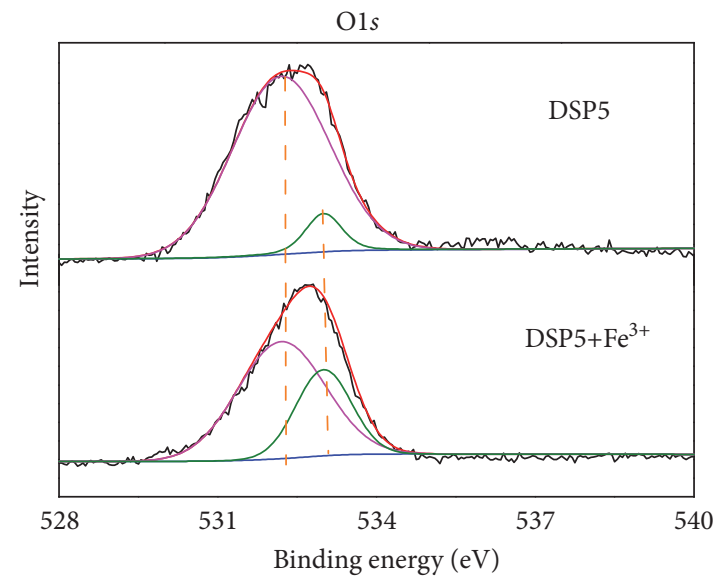

(b)

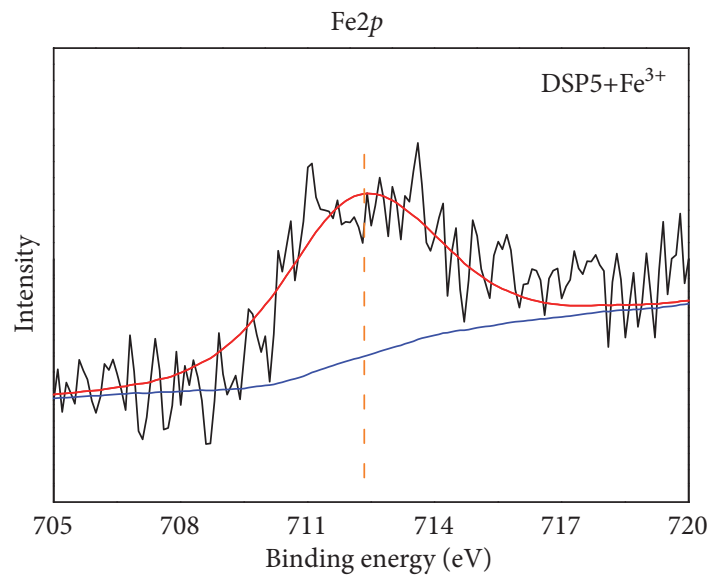

(d)

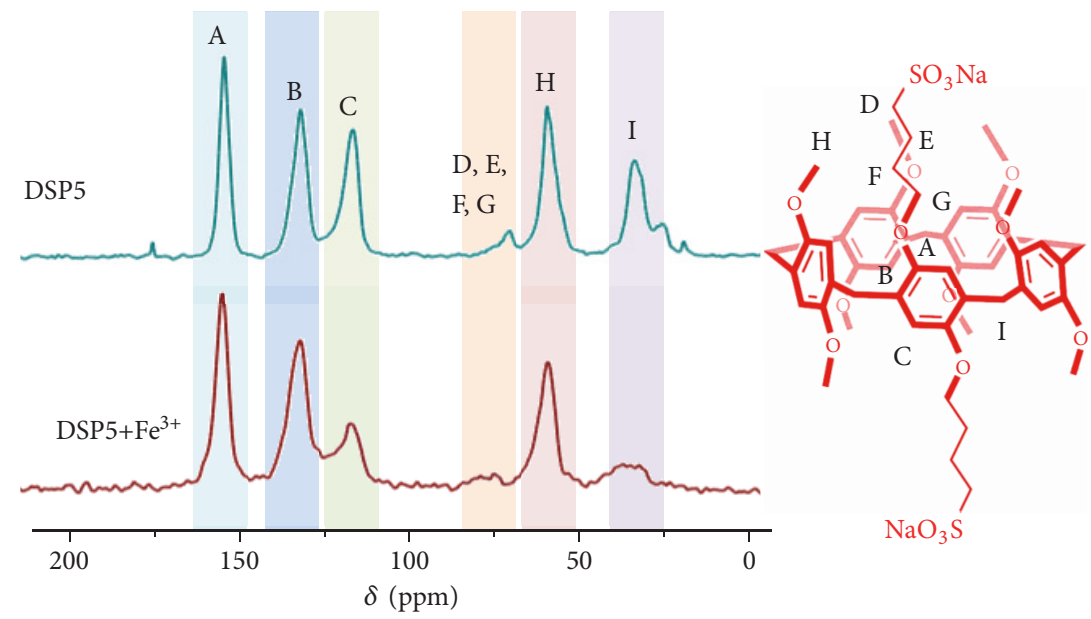

(e)

FIGURE 4: Coordination effect between DSP5 and $\mathrm{Fe}^{3+}$. (a) C1s, (b) O1s, and (c) S2p regions of XPS spectra of DSP5 before (up) and after (down) the addition of $\mathrm{Fe}^{3+}$, and (d) Fe2p regions of XPS spectrum of DSP5 $+\mathrm{Fe}^{3+}$ powder. (e) Solid-state CP-MAS ${ }^{13} \mathrm{C}$ NMR characterization of DSP5 and DSP5 $+\mathrm{Fe}^{3+}$ powder. 
by the coordination effect of DSP 5 and $\mathrm{Fe}^{3+}$. We envision that this new type of supramolecular fluorescent materials will find great applications in various fields, including fluorescent probing, cell imaging, and visualized monitoring of metal ions.

\section{Materials and Methods}

4.1. Materials and Characterization. All reagents were commercially available and used as supplied without further purification. ${ }^{1} \mathrm{H}$ NMR spectra were collected on a temperature-controlled $300 \mathrm{MHz}$ spectrometer. ${ }^{13} \mathrm{C} \mathrm{NMR}$ spectra were recorded on a DMX-500 spectrometer. Solidstate cross-polarization magic angle spinning (CP/MAS) ${ }^{13} \mathrm{C}$ NMR spectra of polymers were measured on a Bruker Digital Avance III HD $400 \mathrm{WB}(400 \mathrm{MHz}) \mathrm{NMR}$ spectrometer at ambient temperature with a magic angle spinning rate of $7.0 \mathrm{kHz}$ internal reference. 2D diffusion-ordered NMR spectroscopy (DOSY) and 2D nuclear Overhauser effect NMR spectroscopy (NOESY) were recorded on a Bruker Avance DMX 600 spectrophotometer. High-resolution electrospray ionization mass spectra (HRESI-MS) were obtained on a Bruker 7-Tesla FT-ICR mass spectrometer equipped with an electrospray source (Billerica, MA, USA). The UVvis spectra were collected via UV-vis spectroscopy on a Shimadzu UV-1800 spectrophotometer. Deionized water, purifying by Experimental Water System (Lab-UV-20), was used in relevant experiments. Scanning electron microscope (SEM) investigations were carried out on a HITACHISU8020 instrument. Dynamic light scattering (DLS) results were obtained on a Zetasizer Nano ZS instrument. The fluorescence experiments were conducted on a RF-5301 spectrofluorometer (Shimadzu Corporation, Japan). The surface compositions of polymers were determined by X-ray photoelectron spectroscopy (XPS) measured on a PREVAC XPS/UPS System using monochromatic Al $K_{\alpha}(1486.7 \mathrm{eV})$ radiation. The time-resolved fluorescence decay curves and absolute fluorescence quantum yields were obtained on a FLS920 instrument (Edinburgh Instrument) with the excitation of $330 \mathrm{~nm}$.

4.2. Syntheses of Compound. The detailed synthesis procedures and characterizations of the studied compound (DSP5, TPE-Q ${ }_{4}$, TPE-Q, and DSNa) can be found in the Supplementary Materials.

4.3. Detection of $\mathrm{Fe}^{3+}$. Experiments were carried out to examine the potential for detecting cations and anions. Firstly, we prepared the DSP5 and TPE- $Q_{4} \subset$ DSP5 aqueous solution $\left(\left[\mathrm{TPE}-\mathrm{Q}_{4}\right]=4 \mu \mathrm{M},[\mathrm{DSP} 5]=1 \mu \mathrm{M}\right) .40 \mu \mathrm{L} \mathrm{M}(\mathrm{Cl})_{\mathrm{x}}$ aqueous solution $\left(\mathrm{M}^{\mathrm{x}+}=\mathrm{Na}^{+}, \mathrm{Fe}^{3+}, \mathrm{Fe}^{2+}, \mathrm{Co}^{2+}, \mathrm{Ni}^{2+}, \mathrm{Cu}^{2+}, \mathrm{Cu}^{+}\right.$, $\mathrm{Zn}^{2+}, \mathrm{Cd}^{2+}, \mathrm{Hg}^{2+}, \mathrm{Mg}^{2+}, \mathrm{K}^{+}, \mathrm{Ca}^{2+}, \mathrm{NH}_{4}{ }^{+}, \mathrm{Pr}^{3+}, \mathrm{Nd}^{3+}, \mathrm{Sm}^{3+}$, $\mathrm{Eu}^{3+}, \mathrm{Gd}^{3+}, \mathrm{Tb}^{3+}, \mathrm{Dy}^{3+}, \mathrm{Ho}^{3+}, \mathrm{Er}^{3+}, \mathrm{Tm}^{3+}, \mathrm{Yb}^{3+}, \mathrm{Lu}^{3+}$, and $\left.\mathrm{Al}^{3+},\left[\mathrm{M}^{\mathrm{x}+}\right]=2 \mathrm{mM}\right)$ and $40 \mu \mathrm{L}(\mathrm{Na})_{\mathrm{y}} \mathrm{N}$ aqueous solution $\left(\mathrm{N}^{\mathrm{y}-}=\mathrm{Cl}^{-}, \mathrm{F}^{-}, \mathrm{OH}^{-}, \mathrm{ClO}^{-}, \mathrm{CH}_{3} \mathrm{COO}^{-}, \mathrm{NO}_{2}{ }^{-}, \mathrm{HPO}_{4}{ }^{2-}\right.$, $\mathrm{H}_{2} \mathrm{PO}_{4}{ }^{-}, \mathrm{PO}_{4}{ }^{3-}, \mathrm{S}_{2} \mathrm{O}_{3}{ }^{2-}, \mathrm{HSO}_{3}{ }^{-}, \mathrm{SO}_{4}{ }^{2-}, \mathrm{S}_{2} \mathrm{O}_{4}{ }^{2-}, \mathrm{HCO}_{3}{ }^{-}$, $\mathrm{CO}_{3}{ }^{2-},\left[\mathrm{N}^{\mathrm{Y}-}\right]=2 \mathrm{mM}$ ) were added into the above TPE- $\mathrm{Q}_{4} \mathrm{C}$ DSP5 solution $(3.96 \mathrm{~mL})$, respectively. Then, the fluorescence of each solution was recorded immediately. Similarly, interference metal ion with the same concentration as $\mathrm{Fe}^{3+}$ was added into the TPE- $\mathrm{Q}_{4} \subset \mathrm{DSP} 5$ solution containing $\mathrm{Fe}^{3+}$ to further investigate the fluorescence recognition of $\mathrm{Fe}^{3+}$. The fluorescence quenching induced by $\mathrm{Fe}^{3+}$ was investigated to confirm whether the developed TPE-Q 4 C DSP5 could be used to quantitative detection of $\mathrm{Fe}^{3+}$. Thus, different volumes of $\mathrm{Fe}^{3+}$ solutions $(1 \mathrm{mM}, 0 \sim 28 \mu \mathrm{L})$ were added into $\mathrm{TPE}_{-} \mathrm{Q}_{4} \subset$ DSP5 solution $(1 \mu \mathrm{M})$ and the fluorescence intensities were recorded, respectively.

4.4. Preparation of DSP5 $+\mathrm{Fe}^{3+}$ Powder. $\mathrm{FeCl}_{3}$ aqueous solution $(1 \mathrm{~mL}, 0.1 \mathrm{mM})$ was added to DSP5 aqueous solution $(0.1$ $\mathrm{mM}, 8 \mathrm{~mL}$ ) and the precipitates were generated immediately. Let the mixture stood for about $24 \mathrm{~h}$ and the precipitates were isolated by centrifuge at $10000 \mathrm{rpm}$. Then the product was washed with deionized water for three times to remove the $\mathrm{FeCl}_{3}$ residue and dried under high vacuum oven to get a brown powder.

\section{Conflicts of Interest}

The authors declare no financial conflicts of interest.

\section{Authors' Contributions}

Conceptualization: Xu Wang, Xiao-Yu Jin, and Ying-Wei Yang. Methodology: Xu Wang, Xiao-Yu Jin, and Ying-Wei Yang. Investigation: $\mathrm{Xu}$ Wang and Xiao-Yu Jin. Writing original draft: Xu Wang and Xin-Yue Lou. Writing review \& editing: Feng Liang and Ying-Wei Yang. Funding acquisition: Ying-Wei Yang. Resources: Feng Liang and Ying-Wei Yang. Supervision: Ying-Wei Yang. All the authors have approved the final version of the manuscript.

\section{Acknowledgments}

This work was supported by the National Natural Science Foundation of China (51673084 and 21871108), the Jilin Province-University Cooperative Construction Project-Special Funds for New Materials (SXGJSF2017-3), and the Jilin University Talents Cultivation Program for financial support.

\section{Supplementary Materials}

Scheme S1. Synthetic route of the targeted compound DSP5. Scheme S2. Synthetic route of the targeted compound TPE$\mathrm{Q}_{4}$. Scheme S3. Synthetic route of the targeted compound TPE-Q. Scheme S4. Synthetic route of the targeted compound DSNa. Figure S1. The study of (a) and (b) association constant $\left(K_{\mathrm{a}}\right)$, and $(\mathrm{c})$ stoichiometric ratio between DSP5 and TPEQ. Figure S2. NOESY spectrum $\left(600 \mathrm{MHz}, \mathrm{D}_{2} \mathrm{O}, 298 \mathrm{~K}\right)$ of a mixture of TPE-Q 4 (1.0 mM) and DSP5 (4.00 mM). Figure S3. Partial NOESY spectrum $\left(600 \mathrm{MHz}, \mathrm{D}_{2} \mathrm{O}, 298 \mathrm{~K}\right)$ of a mixture of TPE-Q 4 (1.0 mM) and DSP5 (4.00 mM). Figure S4. DOSY spectrum $\left(600 \mathrm{MHz}, \mathrm{D}_{2} \mathrm{O}, 298 \mathrm{~K}\right)$ of (a) TPE$\mathrm{Q}_{4}(1.00 \mathrm{mM})$ and $(\mathrm{b})$ a mixture of $\mathrm{TPE}^{-} \mathrm{Q}_{4}(1.0 \mathrm{mM})$ and 
DSP5 (4.00 mM). Figure S5. UV-vis spectra of TPE- $\mathrm{Q}_{4}(1 \mu \mathrm{M})$ in water with different concentrations of DSP5. Figure S6. Fluorescence quenching degrees in competition experiments of TPE- $\mathrm{Q}_{4} \quad$ C DSP5 in water. Front: TPE-Q ${ }_{4}$ C DSP5 with different competing cations; back: TPE- ${ }_{4} \subset$ DSP5 with $\mathrm{Fe}^{3+}$ and the same equiv of cations. (Experimental conditions: $\lambda_{\mathrm{ex}}$ $=330 \mathrm{~nm} ; \lambda_{\mathrm{em}}=475 \mathrm{~nm}$; slit widths: Ex. $5 \mathrm{~nm}$, Em. $5 \mathrm{~nm}$; $298 \mathrm{~K} ;\left[\mathrm{TPE}-\mathrm{Q}_{4}\right]=1 \mu \mathrm{M}$, [DSP5] $=4 \mu \mathrm{M},\left[\mathrm{Fe}^{3+}\right]=20 \mu \mathrm{M}$, [Ions] $=20 \mu \mathrm{M}$ ). Figure S7. Fluorescence quenching degrees and the Stern-Volmer formula constants of TPE- $\mathrm{Q}_{4} \subset$ DSP5 in the presence of different cations and anions in water. 2D plots for all the $\mathrm{M}^{\mathrm{x}+}$ with $\mathrm{Cl}^{-}(\mathrm{a}, \mathrm{c})$ and $\mathrm{Na}^{+}$with $\mathrm{N}^{\mathrm{y}-}$ (b, d). Figure S8. (a) Fluorescence spectra of supramolecular assemblies TPE- $\mathrm{Q}_{4} \subset \mathrm{DSP} 5$ aqueous solution in the presence of increasing amounts of $\mathrm{Fe}^{3+}$; (b) the photograph of the linear range (experimental conditions: $\lambda_{\mathrm{ex}}=330 \mathrm{~nm} ; \lambda_{\mathrm{em}}=$ 475 nm; slit widths: Ex. 5 nm, Em. 5 nm; 298 K; [TPE-Q ${ }_{4}$ ] $=1 \mu \mathrm{M},[\mathrm{DSP} 5]=4 \mu \mathrm{M})$. Figure S9. Fluorescence spectra of TPE-Q4 C DSP5 in the presence of $\mathrm{Fe}^{3+}$ under different $\mathrm{pH}$ values. Figure S10. SEM images of (a) DSP5 $+\mathrm{Fe}^{3+}$ and (b) DSNa+Fe $e^{3+}$; TEM images of (c) DSP5 $+\mathrm{Fe}^{3+}$ and (d) TPE$\mathrm{Q}_{4} \subset \mathrm{DSP} 5+\mathrm{Fe}^{3+}([\mathrm{DSP} 5]=10 \mu \mathrm{M},[\mathrm{DSNa}]=50 \mu \mathrm{M}$, $[$ DSP5 $\left.] /\left[\mathrm{Fe}^{3+}\right]=1: 20,[\mathrm{DSNa}] /\left[\mathrm{Fe}^{3+}\right]=1: 20\right)$. Figure S11. ${ }^{1} \mathrm{H}$ NMR spectra of TPE-Q ${ }_{4}$, TPE-Q 4 C DSP5 and TPE- $Q_{4} \subset$ DSP5 $+\mathrm{Fe}^{3+}\left(\left[\mathrm{TPE}-\mathrm{Q}_{4}\right]=1.00 \mathrm{mM},[\mathrm{DSP} 5]=4.00 \mathrm{mM},\left[\mathrm{Fe}^{3+}\right]\right.$ $\left.=20.00 \mathrm{mM}, 300 \mathrm{MHz}, \mathrm{D}_{2} \mathrm{O}, 298 \mathrm{~K}\right)$. Figure S12. The pictures of (A) TPE- $\mathrm{Q}_{4}$, (B) TPE-Q 4 C DSP5, (C) TPE-Q 4 C DSP5 $+\mathrm{Fe}^{3+}$ and (D) supernatant upon addition of DSP5 again after removing the DSP5 $+\mathrm{Fe}^{3+}$ precipitate. Figure S13. The pictures of (A) TPE-Q , $_{4}$ (B) TPE- $\mathrm{Q}_{4}$ C DSP5, (C) TPE$\mathrm{Q}_{4} \subset \mathrm{DSP} 5+\mathrm{Fe}^{3+}$, and (D) DSP5 $+\mathrm{Fe}^{3+}$ in water (after centrifugation). Figure S14. ${ }^{1} \mathrm{H}$ NMR spectrum $(300 \mathrm{MHz}$, $\mathrm{CDCl}_{3}, 298 \mathrm{~K}$ ) of compound 1 . Figure S15. ${ }^{1} \mathrm{H}$ NMR spectrum (300 MHz, $\mathrm{CDCl}_{3}, 298 \mathrm{~K}$ ) of compound 2. Figure S16. ${ }^{1} \mathrm{H}$ NMR spectrum $\left(300 \mathrm{MHz}, \mathrm{CDCl}_{3}, 298 \mathrm{~K}\right.$ ) of compound 3. Figure S17. ${ }^{13} \mathrm{C}$ NMR spectrum $\left(126 \mathrm{MHz}, \mathrm{D}_{2} \mathrm{O}, 298 \mathrm{~K}\right)$ of DSP5. Figure S18. ${ }^{1} \mathrm{H}$ NMR spectrum $\left(300 \mathrm{MHz}, \mathrm{D}_{2} \mathrm{O}, 298\right.$ $\mathrm{K})$ of DSP5. Figure S19. HRESI-MS m/z $[\mathrm{M}-\mathrm{Na}]^{-}$calcd. for $\mathrm{C}_{51} \mathrm{H}_{60} \mathrm{O}_{16} \mathrm{~S}_{2} \mathrm{Na}^{-}$1015.3215, found 1015.3226. Figure S20. ${ }^{1} \mathrm{H}$ NMR spectrum ( $300 \mathrm{MHz}$, DMSO- $d_{6}, 298 \mathrm{~K}$ ) of compound 4. Figure S21. ${ }^{1} \mathrm{H}$ NMR spectrum $\left(300 \mathrm{MHz}, \mathrm{D}_{2} \mathrm{O}, 298 \mathrm{~K}\right)$ of TPE-Q 4 . Figure S22. ${ }^{1} \mathrm{H}$ NMR spectrum $\left(300 \mathrm{MHz}, \mathrm{CDCl}_{3}\right.$, $298 \mathrm{~K}$ ) of compound 5. Figure S23. ${ }^{1} \mathrm{H}$ NMR spectrum (300 $\mathrm{MHz}, \mathrm{CDCl}_{3}, 298 \mathrm{~K}$ ) of compound 6. Figure S24. ${ }^{1} \mathrm{H}$ NMR spectrum $\left(300 \mathrm{MHz}, \mathrm{CDCl}_{3}, 298 \mathrm{~K}\right)$ of compound 7. Figure S25. ${ }^{1} \mathrm{H}$ NMR spectrum $\left(300 \mathrm{MHz}, \mathrm{DMSO}-d_{6}, 298 \mathrm{~K}\right)$ of TPE-Q. Figure S26. ${ }^{1} \mathrm{H}$ NMR spectrum $\left(300 \mathrm{MHz}, \mathrm{D}_{2} \mathrm{O}, 298\right.$ $\mathrm{K})$ of DSNa. Table S1. Emission lifetimes $(\tau)$, radiative decay rate constant $\left(k_{\text {rad }}\right)$, and nonradiative decay rate constant $\left(k_{\text {nrad }}\right)$ of bare TPE-Q 4 and TPE-Q ${ }_{4} \quad$ C DSP5 in water. (Supplementary Materials)

\section{References}

[1] H. Wang, X. Ji, Z. Li, and F. Huang, "Fluorescent supramolecular polymeric materials," Advanced Materials, vol. 29, no. 14, p. 1606117, 2017.
[2] X. Lou and Y. Yang, "Manipulating aggregation-induced emission with supramolecular macrocycles," Advanced Optical Materials, vol. 6, no. 22, p. 1800668, 2018.

[3] J. Luo, Z. Xie, J. W. Lam et al., "Aggregation-induced emission of 1-methyl-1,2,3,4,5-pentaphenylsilole," Chemical Communications, no. 18, pp. 1740-1741, 2001.

[4] Y. Hong, J. W. Lam, and B. Z. Tang, "Aggregation-induced emission: phenomenon, mechanism and applications," Chemical Communications, no. 29, pp. 4332-4353, 2009.

[5] J. Mei, N. L. C. Leung, R. T. K. Kwok, J. W. Y. Lam, and B. Z. Tang, "Aggregation-induced emission: together we shine, united we soar!," Chemical Reviews, vol. 115, no. 21, pp. 11718-11940, 2015.

[6] G. Liang, J. W. Lam, W. Qin, J. Li, N. Xie, and B. Z. Tang, "Molecular luminogens based on restriction of intramolecular motions through host-guest inclusion for cell imaging," Chemical Communication, vol. 50, no. 14, pp. 1725-1727, 2014.

[7] N. Song, D. Chen, Y. Qiu et al., "Stimuli-responsive blue fluorescent supramolecular polymers based on a pillar[5] arene tetramer," Chemical Communications, vol. 50, no. 60 , pp. 82318234, 2014.

[8] N. Song, D. Chen, M. Xia et al., "Supramolecular assemblyinduced yellow emission of 9,10-distyrylanthracene bridged bis(pillar[5]arene)s," Chemical Communications, vol. 51, no. 25, pp. 5526-5529, 2015.

[9] N. Song, X. Lou, W. Hou, C. Wang, Y. Wang, and Y. Yang, "Pillararene-based fluorescent supramolecular systems: the key role of chain length in gelation," Macromolecular Rapid Communications, vol. 39, no. 24, Article ID e1800593, 2018.

[10] D. Dai, Z. Li, J. Yang et al., "Supramolecular assembly-induced emission enhancement for efficient mercury(II) detection and removal," Journal of the American Chemical Society, vol. 141, no. 11, pp. 4756-4763, 2019.

[11] J. Zhou, B. Hua, L. Shao, H. Feng, and G. Yu, "Host-guest interaction enhanced aggregation-induced emission and its application in cell imaging," Chemical Communications, vol. 52, no. 33, pp. 5749-5752, 2016.

[12] G. Yu, G. Tang, and F. Huang, "Supramolecular enhancement of aggregation-induced emission and its application in cancer cell imaging," Journal of Materials Chemistry C, vol. 2, no. 32, pp. 6609-6617, 2014.

[13] Y. Sun, J. Wang, and Y. Yao, "The first water-soluble pillar[5] arene dimer: synthesis and construction of a reversible fluorescent supramolecular polymer network in water," Chemical Communications, vol. 53, no. 1, pp. 165-167, 2017.

[14] H. Cheng, Z. Li, Y. Huang, L. Liu, and H. Wu, "Pillararene-based aggregation-induced-emission-active supramolecular system for simultaneous detection and removal of mercury(II) in water," ACS Applied Materials \& Interfaces, vol. 9, no. 13, pp. 11889-11894, 2017.

[15] X. Ma, J. Wang, H. Tian et al., "Assembling-induced emission: an efficient approach for amorphous metal-free organic emitting materials with room-temperature phosphorescence," Accounts of Chemical Research, vol. 52, no. 3, pp. 738-748, 2019.

[16] G. Yu, R. Zhao, D. Wu et al., "Pillar[5]arene-based amphiphilic supramolecular brush copolymers: fabrication, controllable self-assembly and application in self-imaging targeted drug delivery," Polymer Chemistry, vol. 7, no. 40, pp. 6178-6188, 2016.

[17] P. Wang, X. Yan, and F. Huang, "Host-guest complexation induced emission: a pillar[6]arene-based complex with intense fluorescence in dilute solution," Chemical Communications, vol. 50, no. 39, pp. 5017-5019, 2014. 
[18] R. Chen, H. Jiang, H. Gu et al., "A pH-responsive fluorescent [5]pseudorotaxane formed by self-assembly of cationic watersoluble pillar[5]arenes and a tetraphenylethene derivative," Chemical Communications, vol. 51, no. 61, pp. 12220-12223, 2015.

[19] X. Jin, N. Song, X. Wang, C. Wang, Y. Wang, and Y. Yang, "Monosulfonicpillar[5]arene: synthesis, characterization, and complexation with tetraphenylethene for aggregation-induced emission," Scientific Reports, vol. 8, no. 1, p. 4035, 2018.

[20] N. L. Strutt, H. Zhang, S. T. Schneebeli, and J. F. Stoddart, "Functionalizing Pillar[n]arenes," Accounts of Chemical Research, vol. 47, no. 8, pp. 2631-2642, 2014.

[21] T. Kakuta, T. Yamagishi, and T. Ogoshi, "Stimuli-responsive supramolecular assemblies constructed from pillar[n]arenes," Accounts of Chemical Research, vol. 51, no. 7, pp. 1656-1666, 2018.

[22] Y.-W. Yang, Y.-L. Sun, and N. Song, "Switchable host-guest systems on surfaces," Accounts of Chemical Research, vol. 47, no. 7, pp. 1950-1960, 2014.

[23] Q. Yao, B. Lü, C. Ji, Y. Cai, and M. Yin, "Supramolecular host-guest system as ratiometric $\mathrm{Fe} 3+$ ion sensor based on water-soluble Pillar[5]arene," ACS Applied Materials \& Interfaces, vol. 9, no. 41, pp. 36320-36326, 2017.

[24] L. Gao, M. Li, S. Ehrmann, Z. Tu, and R. Haag, "Positively charged nanoaggregates based on zwitterionic pillar[5]arene that combat planktonic bacteria and disrupt biofilms," Angewandte Chemie International Edition, vol. 58, no. 11, pp. 36453649, 2019.

[25] X. Hu, X. Liu, W. Zhang et al., "Controllable construction of biocompatible supramolecular micelles and vesicles by watersoluble phosphate Pillar[5,6]arenes for selective anti-cancer drug delivery," Chemistry of Materials, vol. 28, no. 11, pp. 37783788, 2016.

[26] B. Li, Z. Meng, Q. Li et al., "A pH responsive complexationbased drug delivery system for oxaliplatin," Chemical Science, vol. 8, no. 6, pp. 4458-4464, 2017.

[27] M. Ni, N. Zhang, W. Xia et al., "Dramatically promoted swelling of a hydrogel by Pillar[6]arene-ferrocene complexation with multistimuli responsiveness," Journal of the American Chemical Society, vol. 138, no. 20, pp. 6643-6649, 2016.

[28] N. Song and Y. Yang, "Molecular and supramolecular switches on mesoporous silica nanoparticles," Chemical Society Reviews, vol. 44, no. 11, pp. 3474-3504, 2015.

[29] M.-X. Wu, J. Gao, F. Wang et al., "Multistimuli responsive coreshell nanoplatform constructed from Fe3O4 @MOF equipped with pillar[6] arene nanovalves," Small, vol. 14, no. 17, Article ID el704440, 2018.

[30] J. Chen, Q. Lin, Y. Zhang, H. Yao, and T. Wei, "Pillararene-based fluorescent chemosensors: recent advances and perspectives," Chemical Communications, vol. 53, no. 100, pp. 13296-13311, 2017.

[31] M. Volmer, M. Stratmann, and H. Viefhaus, "Electrochemical and electron spectroscopic investigations of iron surfaces modified with thiols," Surface and Interface Analysis, vol. 16, no. 1-12, pp. 278-282, 1990.

[32] Y. I. Kim and W. E. Hatfield, "Electrical, magnetic and spectroscopic properties of tetrathiafulvalene charge transfer compounds with iron, ruthenium, rhodium and iridium halides," Inorganica Chimica Acta, vol. 188, no. 1, pp. 15-24, 1991. 